\title{
A Relaxed Greedy Block Kaczmarz Method for Solving Large Consistent Linear Systems
}

\author{
Yimou Liao', Feng Yin 1,2*, Guangxin Huang3 \\ ${ }^{1}$ College of Mathematics and Statistics, Sichuan University of Science \& Engineering, Zigong, China \\ ${ }^{2}$ Sichuan Province University Key Laboratory of Bridge Non-destruction Detecting and Engineering Computing, Zigong, China \\ ${ }^{3}$ College of Mathematics and Physics, Chengdu University of Technology, Chengdu, China \\ Email: fyinsuse@163.com, liaoyimou596@163.com, huangx@cdut.edu.cn
}

How to cite this paper: Liao, Y.M., Yin, F. and Huang, G.X. (2021) A Relaxed Greedy Block Kaczmarz Method for Solving Large Consistent Linear Systems. Journal of Applied Mathematics and Physics, 9, 3032-3044. https://doi.org/10.4236/jamp.2021.912196

Received: October 29, 2021

Accepted: December 12, 2021

Published: December 15, 2021

Copyright $\odot 2021$ by author(s) and Scientific Research Publishing Inc. This work is licensed under the Creative Commons Attribution International License (CC BY 4.0).

http://creativecommons.org/licenses/by/4.0/

\begin{abstract}
Many problems in science and engineering require solving large consistent linear systems. This paper presents a relaxed greedy block Kaczmarz method (RGBK) and an accelerated greedy block Kaczmarz method (AGBK) for solving large-size consistent linear systems. The RGBK algorithm extends the greedy block Kaczmarz algorithm (GBK) presented by Niu and Zheng in [1] by introducing a relaxation parameter to the iteration formulation of GBK, and the AGBK algorithm uses different iterative update rules to minimize the running time. The convergence of the RGBK is proved and a method to determine an optimal parameter is provided. Several examples are presented to show the effectiveness of the proposed methods for overdetermined and underdetermined consistent linear systems with dense and sparse coefficient matrix.
\end{abstract}

\section{Keywords}

Linear Consistent Systems, Convergence Properties, Relaxed Greedy Block Kaczmarz

\section{Introduction}

We are concerned with the solution of the large consistent linear system

$$
A x=b,
$$

where $\boldsymbol{A} \in \mathbb{R}^{m \times n}$, and $b \in \mathbb{R}^{m}$. The Kaczmarz method in [2] is possible one of the most popular, simple while efficient algorithms for solving (1). It was revised to be applied to image reconstruction in [3], which is called algebraic reconstruction technique, and has a large range of fields of applications such as image reconstruction in computerized tomography [4] [5] [6] and parallel computing [7]. 
There are many extended Kaczmarz algorithms [8]-[16] developed to solve (1). Strohmer and Vershynin in [17] introduced a randomized Kaczmarz method (RK) for consistent overdetermined systems (1). The RK method has a convergence bound with the expected exponential convergence, which was called linear convergence. Zhang [18] proposed an improved greedy Kaczmarz (GK) method for solving (1). Bai and $\mathrm{Wu}$ in [19] presented a greedy randomized Kaczmarz algorithm (GRK) for (1) when the system is consistent. In each step of iteration, GRK is based on a probability criterion trying to grasp larger entries of the residual vector. Bai and $\mathrm{Wu}$ [20] further developed a relaxed GRK method for large sparse Equations (1). Due to its fast convergence, the block method [21] [22] [23] [24] has also been extensively developed in linear or nonlinear optimization problems. Recently, Liu and Zheng in [1] presented a greedy block Kaczmarz algorithm (GBK) with the iteration

$$
x_{k+1}=x_{k}+A_{\mathcal{J}_{k}}^{\dagger}\left(b_{\mathcal{J}_{k}}-\boldsymbol{A}_{\mathcal{J}_{k}} x_{k}\right),
$$

where the index of the selected row

$$
\mathcal{J}_{k}=\left\{i_{k}|| b_{i_{k}}-\left.\boldsymbol{A}^{\left(i_{k}\right)} x_{k}\right|^{2} \geq \varepsilon_{k}\left\|\boldsymbol{A}^{\left(i_{k}\right)}\right\|^{2}\right\}
$$

with the parameter

$$
\varepsilon_{k}=\eta \max _{1 \leq i \leq m}\left\{\frac{\left|b_{i}-\boldsymbol{A}^{(i)} x_{k}\right|^{2}}{\left\|\boldsymbol{A}^{(i)}\right\|_{2}^{2}}\right\} .
$$

$\boldsymbol{A}_{\mathcal{J}_{k}}$ stands for the submatrix $\boldsymbol{A}\left(\mathcal{J}_{k},:\right)$ of $\boldsymbol{A}, b_{i}$ represents the $i$ th element of the vector $b, A^{(i)}$ denotes the ith row of $\boldsymbol{A}$ and $\boldsymbol{A}^{\dagger}$ denotes the MoorePenrose pseudoinverse of $\boldsymbol{A}$.

In this paper, based on the GBK method in [1], we develop a new relaxed greedy block Kaczmarz algorithm (RGBK) for (1). RGBK extends the GBK algorithm by introducing a relaxed parameter. The convergence of the algorithm is provided and the optimal relaxed parameter is discussed. The rest of this paper is organized as follows. In Section 2, a new relaxed greedy block Kaczmarz algorithm is presented. The convergence is provided and the optimal relaxed parameter is determined. Several types of examples are shown in Section 3, including the overdetermined or underdetermined systems with dense and sparse coefficient matrices. Some conclusions are drawn in Section 4.

At the end of this section, we introduce some mathematical symbols that will be used. For a matrix $\boldsymbol{Q} \in \mathbb{R}^{m \times n}, \boldsymbol{Q}^{(i)}$ denotes the $i$ th row vector of $\boldsymbol{Q} \cdot \boldsymbol{Q}_{\mathcal{J}_{k}}$ stands for the submatrix $\boldsymbol{Q}\left(\mathcal{J}_{k},:\right)$ of $\boldsymbol{Q}$, where $\mathcal{J}_{k}$ is an index set composed of positive integers not exceeding $m$, and $m$ is the number of rows of $\boldsymbol{Q}$. Let $\delta_{\min }(\boldsymbol{Q})$ and $\delta_{\max }(\boldsymbol{Q})$ be the maximum and minimum positive singular values of $\boldsymbol{Q}$, respectively. $\|\boldsymbol{Q}\|_{2}^{2}$ and $\|\boldsymbol{Q}\|_{F}^{2}=\sum_{i=1}^{m} \sum_{j=1}^{n}\left|q_{i j}\right|^{2}$ are the spectral norm and Frobenius norm, respectively. For any vector $p \in \mathbb{R}^{m}, p_{i}$ represents the ith component of $p$. 


\section{The Relaxed Greedy Block Kaczmarz Algorithm}

Replacing the left side $x_{k+1}$ in (2) with the combination of $x_{k}$ and $x_{k+1}$ in (2) by introducing a relaxed parameter $\lambda \in(0,2)$, we have

$$
x_{k+1}=(1-\lambda) x_{k}+\lambda\left(x_{k}+\boldsymbol{A}_{\mathcal{J}_{k}}^{\dagger}\left(b_{\mathcal{J}_{k}}-\boldsymbol{A}_{\mathcal{J}_{k}} x_{k}\right)\right) \text {. }
$$

Thus

$$
x_{k+1}=x_{k}+\lambda A_{J_{k}}^{\dagger}\left(b_{J_{k}}-A_{J_{k}} x_{k}\right) .
$$

The method presented by (6) is called a relaxed greedy block Kaczmarz algorithm, which is abbreviated by RGBK. Algorithm 1 summarizes the RGBK algorithm.

We remark that the iteration formulation (6) reduces to (2) when $\lambda=1$, thus the GBK method in [1] is a special case of Algorithm 1.

The results below give the convergence of Algorithm 1.

Theorem 1. Assume the linear system (1) is consistent. The iterative sequence $\left\{x_{k}\right\}$ generated by Algorithm 1 converges to the minimum norm solution $x^{*}=A^{\dagger} b \quad$ of $(1)$. Moreover, it holds that

$$
\left\|x_{k+1}-x^{*}\right\|_{2}^{2} \leq\left[1-\phi_{k}(\lambda, \eta) \frac{\delta_{\min }^{2}(\boldsymbol{A})}{\|\boldsymbol{A}\|_{F}^{2}}\right] \cdot\left\|x_{k}-x^{*}\right\|_{2}^{2}, \quad k=0,1, \cdots,
$$

where $\phi_{k}(\lambda, \eta)=\eta \lambda(2-\lambda) \frac{\left\|\boldsymbol{A}_{\mathcal{J}_{k}}\right\|_{F}^{2}}{\sigma_{\max }^{2}\left(\boldsymbol{A}_{\mathcal{J}_{k}}\right)},\|\boldsymbol{A}\|_{F}$ denotes the Frobenius norm of $\boldsymbol{A}, \delta_{\max }(\boldsymbol{A})$ and $\delta_{\min }(\boldsymbol{A})$ are the maximum and minimum positive singular values of $\boldsymbol{A}$, respectively.

Proof. Let $e_{k}=x_{k}-x^{*}$, where $x^{*}$ satisfies $\boldsymbol{A}_{\mathcal{J}_{k}} x^{*}=b_{J_{k}}$, then we have from (6) that

$$
\begin{aligned}
\left\|e_{k+1}\right\|_{2}^{2} & =\left\|e_{k}+\lambda \boldsymbol{A}_{\mathcal{J}_{k}}^{\dagger}\left(b_{\mathcal{J}_{k}}-\boldsymbol{A}_{\mathcal{J}_{k}} x_{k}\right)\right\|_{2}^{2}=\left\|\left(\boldsymbol{I}-\lambda \boldsymbol{A}_{\mathcal{J}_{k}}^{\dagger} \boldsymbol{A}_{\mathcal{J}_{k}}\right) e_{k}\right\|_{2}^{2} \\
& =\left\|e_{k}\right\|_{2}^{2}-\lambda(2-\lambda)\left\|\boldsymbol{A}_{\mathcal{J}_{k}}^{\dagger} \boldsymbol{A}_{\mathcal{J}_{k}} e_{k}\right\|_{2}^{2} .
\end{aligned}
$$

According to the definition of $\mathcal{J}_{k}$ at Step 3 in Algorithm 1 and the fact that $\delta_{\min }^{2}\left(\boldsymbol{A}_{\mathcal{J}_{k}}^{\dagger}\right)=\delta_{\max }^{-2}\left(\boldsymbol{A}_{\mathcal{J}_{k}}\right)$, we have that

Algorithm 1. A relaxed greedy block Kaczmarz algorithm (RGBK).

Input: $\boldsymbol{A}, b, x_{0}$ parameter $\eta \in(0,1]$ and $\lambda \in(0,2)$.

Output: the approximation solution $x_{k}$ of (1)

for $k=0,1, \cdots$ do until termination criterion is satisfied; do

Compute $\varepsilon_{k}$ by (4);

Determine the control index set $\mathcal{J}_{k}$ by (3);

Update $x_{k+1}$ by (6).

end for 


$$
\begin{aligned}
\left\|\boldsymbol{A}_{\mathcal{J}_{k}}^{\dagger} \boldsymbol{A}_{\mathcal{J}_{k}} e_{k}\right\|_{2}^{2} & \geq \delta_{\min }^{2}\left(\boldsymbol{A}_{\mathcal{J}_{k}}^{\dagger}\right)\left\|\boldsymbol{A}_{\mathcal{J}_{k}} e_{k}\right\|_{2}^{2}=\delta_{\min }^{2}\left(\boldsymbol{A}_{\mathcal{J}_{k}}^{\dagger}\right) \sum_{i_{k} \in \mathcal{J}_{k}}\left|\boldsymbol{A}^{\left(i_{k}\right)} e_{k}\right|^{2} \\
& \geq \delta_{\min }^{2}\left(\boldsymbol{A}_{\mathcal{J}_{k}}^{\dagger}\right) \sum_{i_{k} \in \mathcal{J}_{k}} \varepsilon_{k}\left\|\boldsymbol{A}^{\left(i_{k}\right)}\right\|^{2}=\frac{\left\|\boldsymbol{A}_{\mathcal{J}_{k}}\right\|_{F}^{2}}{\delta_{\max }^{2}\left(\boldsymbol{A}_{\mathcal{J}_{k}}\right)} \varepsilon_{k} .
\end{aligned}
$$

For $k=1,2, \cdots$, it holds that

$$
\left\|b-\boldsymbol{A} x_{k}\right\|_{2}^{2}=\sum_{i=1}^{m} \frac{\left|b_{i}-\boldsymbol{A}^{(i)} x_{k}\right|^{2}}{\left\|\boldsymbol{A}^{(i)}\right\|_{2}^{2}}\left\|\boldsymbol{A}^{(i)}\right\|_{2}^{2} \leq \max _{1 \leq i \leq m} \frac{\left|b_{i}-\boldsymbol{A}^{(i)} x_{k}\right|^{2}}{\left\|\boldsymbol{A}^{(i)}\right\|_{2}^{2}}\|\boldsymbol{A}\|_{F}^{2},
$$

thus the constant $\varepsilon_{k}$ at step 2 of Algorithm 1 becomes

$$
\begin{aligned}
\varepsilon_{k} & =\eta \max _{1 \leq i \leq m} \frac{\left|b_{i}-\boldsymbol{A}^{(i)} x_{k}\right|^{2}}{\left\|\boldsymbol{A}^{(i)}\right\|_{2}^{2}} \geq \eta \frac{\left\|b-\boldsymbol{A} x_{k}\right\|_{2}^{2}}{\|\boldsymbol{A}\|_{F}^{2}} \\
& =\eta \frac{\left\|\boldsymbol{A} x^{*}-\boldsymbol{A} x_{k}\right\|_{2}^{2}}{\|\boldsymbol{A}\|_{F}^{2}} \geq \eta \frac{\delta_{\min }^{2}(\boldsymbol{A})}{\|\boldsymbol{A}\|_{F}^{2}}\left\|e_{k}\right\|_{2}^{2} .
\end{aligned}
$$

Thus (8) together with (9) and (10) implies (7). This completes the proof.

We derive easily the results below from Theorem 1 .

Corollary 1. Let $M(\lambda, \eta)=\max _{0 \leq j \leq k-1} \phi_{j}(\lambda, \eta)$. Under the conditions of Theorem 1 , we obtain an upper bound of error

$$
\left\|x_{k}-x^{*}\right\|_{2}^{2} \leq\left[1-M(\lambda, \eta) \frac{\delta_{\min }^{2}(\boldsymbol{A})}{\|\boldsymbol{A}\|_{F}^{2}}\right]^{k} \cdot\left\|x_{0}-x^{*}\right\|_{2}^{2} .
$$

Proof. Using (7) iteratively for $k=1, \cdots$, we have (11) with the definition of $M(\lambda, \eta)$.

The upper bound of error below is independent of $\mathcal{J}_{k}$.

Corollary 2. Under the conditions of Theorem 1, (7) becomes

$$
\left\|x_{k+1}-x^{*}\right\|_{2}^{2} \leq\left[1-\eta \lambda(2-\lambda) \frac{\delta_{\min }^{2}(\boldsymbol{A})}{\|\boldsymbol{A}\|_{F}^{2}}\right] \cdot\left\|x_{k}-x^{*}\right\|_{2}^{2} .
$$

Proof. We notice that

$$
\left\|\boldsymbol{A}_{\mathcal{J}_{k}}\right\|_{F}^{2}=\sum_{i=1}^{\left|\mathcal{J}_{k}\right|} \delta_{i}^{2}\left(\boldsymbol{A}_{\mathcal{J}_{k}}\right) \geq \delta_{\max }^{2}\left(\boldsymbol{A}_{\mathcal{J}_{k}}\right)
$$

thus

$$
\frac{\left\|\boldsymbol{A}_{\mathcal{J}_{k}}\right\|_{F}^{2}}{\delta_{\max }^{2}\left(\boldsymbol{A}_{\mathcal{J}_{k}}\right)} \geq 1
$$

Therefore, (12) results from (12) together with (7).

Remark 1. The RGBK method reduces to the GBK method in [1] when $\lambda=1$. Examples in Section 3 provide a way to determine the optimal relaxed parameter value of $\lambda$ that minimizes the CPU time or the number of iteration for both overdetermined and underdetermined systems. 
Remark 2. Taking into account the limitation of computer memory space, we use Gaussian Kaczmarz method defined in [22] instead of (6), which could avoid the calculation of $\mathbf{A}^{\dagger}$,

$$
x_{k+1}=x_{k}+\lambda \frac{\delta_{k}^{\mathrm{T}}\left(b-\boldsymbol{A} x_{k}\right)}{\left\|\boldsymbol{A}^{\mathrm{T}} \delta_{k}\right\|_{2}^{2}} A^{\mathrm{T}} \delta_{k},
$$

where $\delta_{k}=\sum_{i \in \mathcal{J}_{k}}\left(b_{i}-\boldsymbol{A}^{(i)} x_{k}\right) e_{i}$, this method abbreviated as AGBK.

\section{Numerical Examples}

In this section, we give several examples to show the efficiency of our RGBK and AGBK methods and compare them with GBK in [1]. All experiments are carried out with the MATLAB $2020 \mathrm{~b}$ on a computer with $3.00 \mathrm{GHz}$ processing unit and 16 GB RAM.

We compute the solution of the consistent system (1) with $\boldsymbol{A} \in \mathbb{R}^{m \times n}$ and $b \in \mathbb{R}^{m}$ computed by $b=\boldsymbol{A} x^{*}$, where $x^{*} \in \mathbb{R}^{n}$ denotes the exact solution generated by the MATLAB function randn. Denote the relative solution residuals RSE : $=\left\|x_{k}-x^{*}\right\|_{2}^{2} /\left\|x^{*}\right\|_{2}^{2}$. We define different acceleration ratios as follows,

$$
\text { SU-R }=\frac{\text { CPU of GBK }}{\text { CPU of RGBK }} \text { and } \quad \text { SU-A }=\frac{\text { CPU of GBK }}{\text { CPU of AGBK }} .
$$

To avoid calculating the Moore-Penrose inverse $\boldsymbol{A}^{\dagger}$ when implementing the update (6) in Algorithm 1, we use the CGLS algorithm in [21] to solve a corresponding least-squares problem. Considering the fairness of the three algorithms, all parameters involved in AGBK are the same as RGBK. We set the initial vector $x_{0}=0$ and the termination criterion satisfying RSE $<10^{-6}$ for GBK, RGBK and AGBK in all examples.

Example 4.1. We use this example to illustrate how to determine an optimal parameter $\eta$ in Algorithm 1 for both overdetermined and underdetermined systems (1). We use different parameter $\eta$ ranged from 0.05 to 0.9 to compute the number of iteration (IT) and CPU time (CPU) by Algorithm 1 for the consistent systems (1) with $\boldsymbol{A} \in \mathbb{R}^{2000 \times 1000}$ and $\boldsymbol{A} \in \mathbb{R}^{1000 \times 2000}$, which are randomly dense matrices generated by the MATLAB function randn, respectively, then determine an optimal parameter $\eta$ that minimizes the IT or CPU.

Figure 1 shows the plot of CPU versus $\eta$. From Figure 1, we can choose the optimal parameter $\eta=0.2$, which minimizes CPU by using Algorithm 1 for the consistent systems (1) with $\boldsymbol{A} \in \mathbb{R}^{2000 \times 1000}$ and $\boldsymbol{A} \in \mathbb{R}^{1000 \times 2000}$.

Example 4.2. In this case, we give the same way to determine the optimal relaxation parameters $\lambda$ of RGBK as shown in Figure 2, and strictly abide by the method of controlling variables. We default that GBK and RGBK have the same $\eta$.

Figure 2 shows the plot of IT and CPU versus relaxed parameter $\lambda$. From Figure 2(a) and Figure 2(b), we can choose the optimal relaxed parameter $\lambda_{\text {opt }}=1.2$ and $\lambda_{\text {opt }}=1.3$, which minimizes CPU by using Algorithm 1 for the consistent systems (1) with $\boldsymbol{A} \in \mathbb{R}^{2000 \times 1000}$ and $\boldsymbol{A} \in \mathbb{R}^{1000 \times 2000}$. 

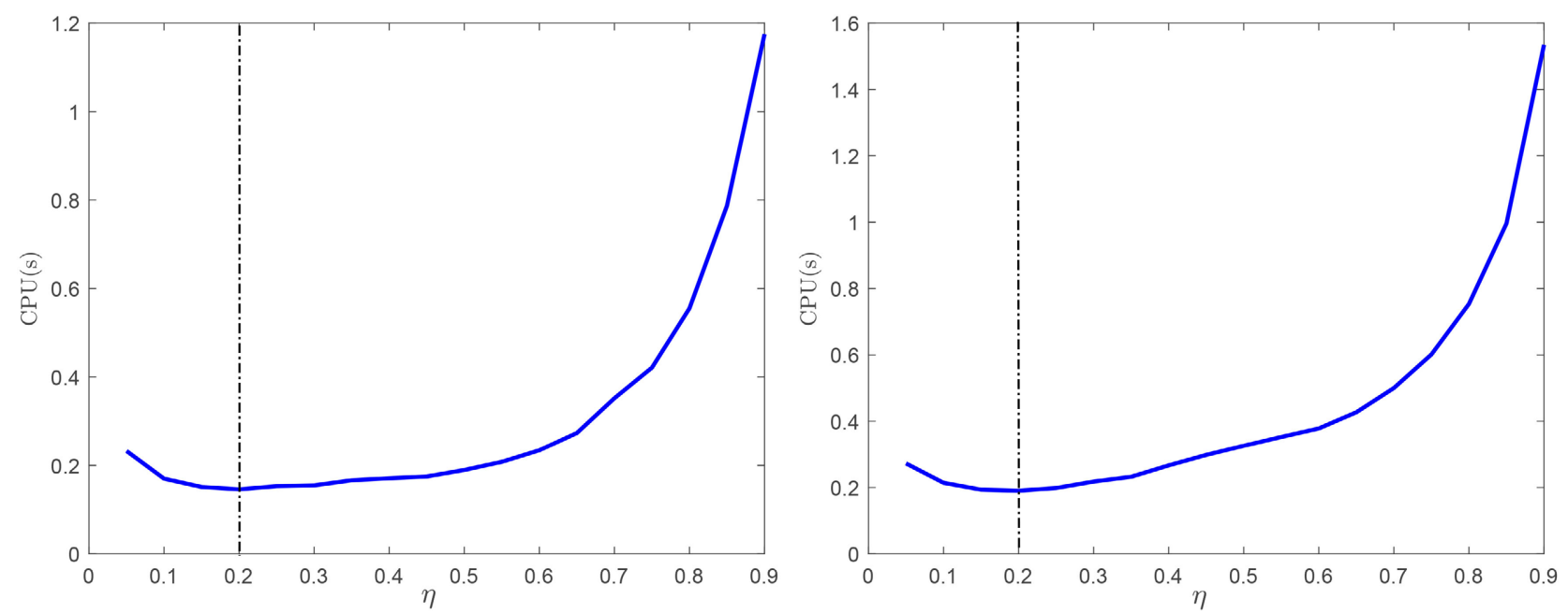

Figure 1. An optimal parameter $(\eta)$ determined by the minimum of CPU for overdetermined $2000 \times 1000$ (left) and underdetermined $1000 \times 2000$ (right) systems in RGBK.
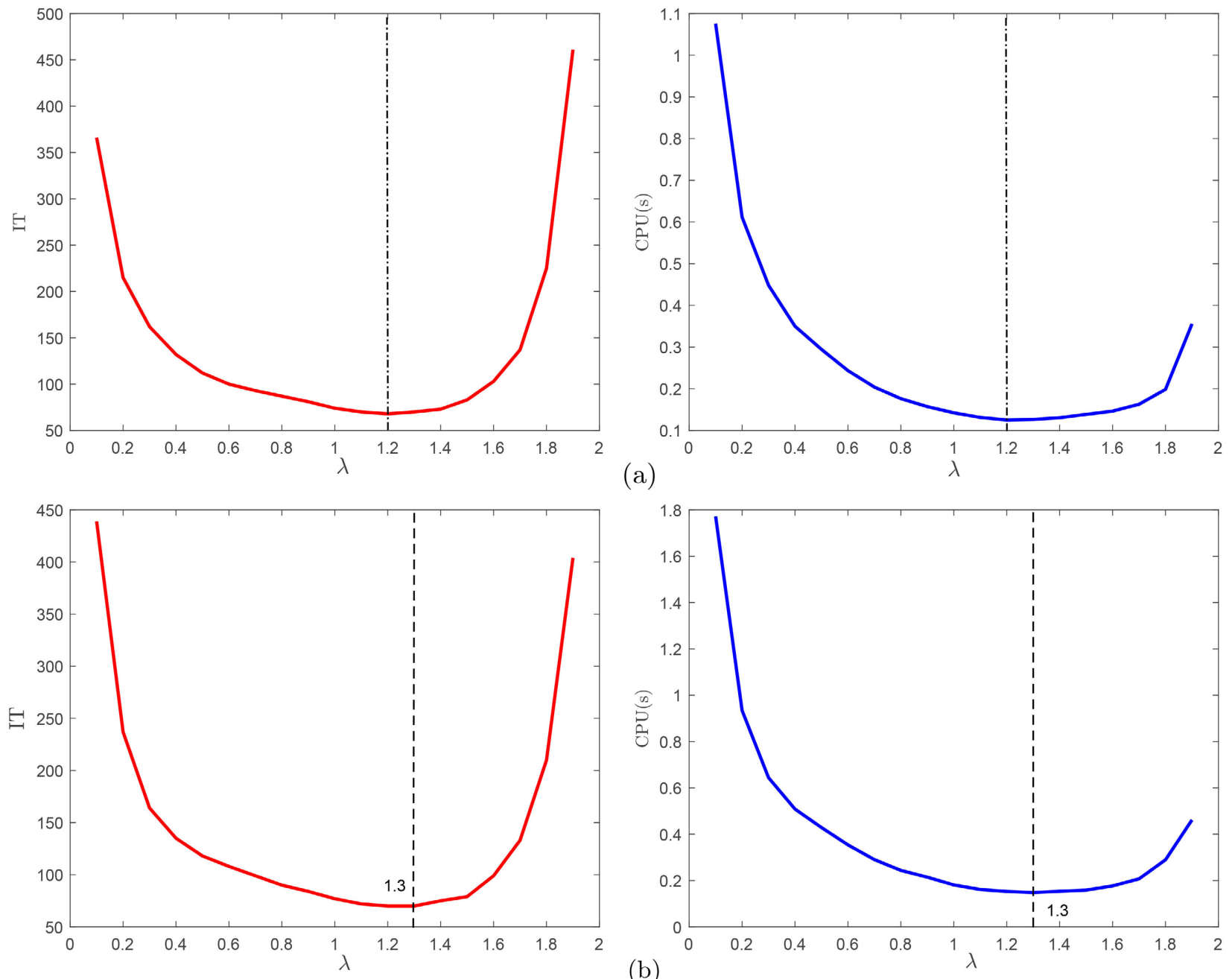

Figure 2. An optimal relaxed parameter $(\lambda)$ determined by the minimum of IT (left) and CPU (right) for overdetermined and underdetermined systems. (a) IT (left) and CPU (right) versus $\lambda$ with $2000 \times 1000$; (b) IT (left) and CPU (right) versus $\lambda$ with $1000 \times 2000$. 
Example 4.3. We compare the convergence of RGBK and AGBK with the optimal relaxed parameter $\lambda_{\text {opt }}$ determined similarly in Example 4.2 with that of GBK algorithm for overdetermined and underdetermined systems (1) with $\boldsymbol{A} \in \mathbb{R}^{m \times n}$ generated by the MATLAB function randn. Table 1 lists IT and CPU(s) of the RGBK and AGBK algorithms with the optimal relaxed parameter $\lambda_{\text {opt }}$ compared with that of GBK algorithm for different overdetermined systems (1) with Gaussian coefficient matrices $\boldsymbol{A}$. The corresponding optimal relaxed parameter computed similarly in Example 4.2 is $\lambda_{\text {opt }}=1.2,1.2,1.3,1.2,1.2,1.2,1.25$, respectively.

Table 2 shows similar results for different underdetermined Gaussian systems (1) with $\boldsymbol{A}$. From Table 1 and Table 2, we can see the advantages of our proposed RGBK and AGBK methods over GBK algorithm.

Example 4.4. We use the RGBK and AGBK methods and compare them with GBK to solve systems (1) with sparse coefficient matrix $\boldsymbol{A}$ from the Florida sparse matrix collection in [25]. Table 3 summarizes the different sparse systems and its density and condition number Cond $(\boldsymbol{A})$, where the density of $\boldsymbol{A}$ means the ratio of the number of the nonzero elements of $A$ to the total number of the elements of $\boldsymbol{A}$.

Table 4 lists IT and CPU(s) of the RGBK and AGBK algorithms with the optimal

Table 1. Performance of GBK, RGBK, AGBK for overdetermined systems.

\begin{tabular}{|c|c|c|c|c|c|}
\hline \multirow{2}{*}{ method } & \multirow{2}{*}{ Index } & \multicolumn{4}{|c|}{$m \times n$} \\
\hline & & $3000 \times 1000$ & $4000 \times 1000$ & $5000 \times 1000$ & $3000 \times 2000$ \\
\hline \multirow[t]{2}{*}{$\operatorname{GBK}(\eta)$} & IT & $37(0.2)$ & $26(0.2)$ & $24(0.25)$ & $159(0.15)$ \\
\hline & $\mathrm{CPU}$ & 0.1064 & 0.0904 & 0.0824 & 1.0852 \\
\hline \multirow[t]{2}{*}{$\operatorname{RGBK}(\lambda)$} & IT & $34(1.2)$ & $24(1.2)$ & $23(1.3)$ & 149 (1.3) \\
\hline & CPU & 0.0884 & 0.0790 & 0.0715 & 0.9881 \\
\hline \multirow[t]{2}{*}{ AGBK } & IT & 36 & 25 & 25 & 158 \\
\hline & $\mathrm{CPU}$ & 0.0587 & 0.0538 & 0.0635 & 0.4778 \\
\hline SU-R & & 1.2039 & 1.1448 & 1.1542 & 1.0983 \\
\hline \multirow[t]{2}{*}{ SU-A } & & 1.8146 & 1.7943 & 1.2983 & 2.2713 \\
\hline & & $4000 \times 2000$ & $5000 \times 2000$ & $4000 \times 3000$ & $5000 \times 3000$ \\
\hline \multirow[t]{2}{*}{$\operatorname{GBK}(\eta)$} & IT & $71(0.15)$ & $43(0.15)$ & $313(0.2)$ & $121(0.15)$ \\
\hline & CPU & 0.6024 & 0.4370 & 3.8447 & 1.9698 \\
\hline \multirow[t]{2}{*}{$\operatorname{RGBK}(\lambda)$} & IT & $66(1.2)$ & $42(1.2)$ & $290(1.2)$ & $115(1.25)$ \\
\hline & $\mathrm{CPU}$ & 0.5577 & 0.4128 & 3.3780 & 1.7867 \\
\hline \multirow[t]{2}{*}{ AGBK } & IT & 69 & 44 & 293 & 120 \\
\hline & $\mathrm{CPU}$ & 0.2730 & 0.2149 & 1.7092 & 0.8857 \\
\hline SU-R & & 1.0801 & 1.0659 & 1.1380 & 1.1025 \\
\hline SU-A & & 2.2061 & 2.0474 & 2.2491 & 2.2241 \\
\hline
\end{tabular}


Table 2. Performance of GBK, RGBK, AGBK for underdetermined systems.

\begin{tabular}{|c|c|c|c|c|c|}
\hline \multirow{2}{*}{ name } & \multirow{2}{*}{ Index } & \multicolumn{4}{|c|}{$m \times n$} \\
\hline & & $1000 \times 3000$ & $1000 \times 4000$ & $1000 \times 5000$ & $2000 \times 3000$ \\
\hline \multirow[t]{2}{*}{$\operatorname{GBK}(\eta)$} & IT & $35(0.15)$ & $23(0.1)$ & $20(0.1)$ & $163(0.15)$ \\
\hline & $\mathrm{CPU}$ & 0.1488 & 0.1392 & 0.1487 & 1.2579 \\
\hline \multirow[t]{2}{*}{$\operatorname{RGBK}(\lambda)$} & IT & $32(1.25)$ & $22(1.2)$ & $19(1.3)$ & $150(1.3)$ \\
\hline & $\mathrm{CPU}$ & 0.1231 & 0.1334 & 0.1418 & 1.0974 \\
\hline \multirow[t]{2}{*}{ AGBK } & IT & 33 & 22 & 19 & 163 \\
\hline & $\mathrm{CPU}$ & 0.0532 & 0.0434 & 0.0457 & 0.4838 \\
\hline SU-R & & 1.2087 & 1.0431 & 1.0487 & 1.1463 \\
\hline \multirow[t]{2}{*}{ SU-A } & & 2.7982 & 3.2101 & 3.2541 & 2.6001 \\
\hline & & $2000 \times 4000$ & $2000 \times 5000$ & $3000 \times 4000$ & $3000 \times 5000$ \\
\hline \multirow[t]{2}{*}{$\operatorname{GBK}(\eta)$} & IT & $65(0.1)$ & $49(0.15)$ & $299(0.15)$ & $142(0.2)$ \\
\hline & CPU & 0.7464 & 0.6181 & 4.3543 & 2.3230 \\
\hline \multirow[t]{2}{*}{$\operatorname{RGBK}(\lambda)$} & IT & $63(1.4)$ & $44(1.25)$ & $280(1.3)$ & $132(1.4)$ \\
\hline & $\mathrm{CPU}$ & 0.7112 & 0.5401 & 3.9027 & 1.9067 \\
\hline \multirow[t]{2}{*}{ AGBK } & IT & 71 & 45 & 294 & 145 \\
\hline & $\mathrm{CPU}$ & 0.2719 & 0.2164 & 1.7181 & 1.0346 \\
\hline SU-R & & 1.0496 & 1.1443 & 1.1157 & 1.2183 \\
\hline SU-A & & 2.7454 & 2.8555 & 2.5344 & 2.2452 \\
\hline
\end{tabular}

Table 3. The properties of different sparse matrices.

\begin{tabular}{cccccc}
\hline name & ash-958 & Trefethen-700 & relat6 & flower-5-1 & stat96v5 \\
\hline$m \times n$ & $958 \times 292$ & $700 \times 700$ & $2340 \times 157$ & $211 \times 201$ & $2307 \times 75,779$ \\
\hline Density & $0.68 \%$ & $2.58 \%$ & $2.20 \%$ & $1.42 \%$ & $0.13 \%$ \\
Full rank & Yes & Yes & No & No & No \\
Cond(A) & 3.20 & $4.71 \mathrm{e}+3$ & Inf & $2.00 \mathrm{e}+16$ & $1.2609 \mathrm{e}+17$ \\
\hline
\end{tabular}

Table 4. Performance of GBK, RGBK, AGBK for overdetermined systems.

\begin{tabular}{ccccccc}
\hline name & & ash958 & Trefethen-700 & relat6 & flower-5-1 & stat96v5 \\
\hline GBK $(\eta)$ & IT & $25(0.25)$ & $468(0.1)$ & $116(0.25)$ & $242(0.25)$ & $55(0.3)$ \\
& CPU & 0.0008 & 0.0219 & 0.0064 & 0.0036 & 0.1006 \\
\hline RGBK $(\lambda)$ & IT & $21(0.9)$ & $401(1.2)$ & $115(0.9)$ & $214(1.2)$ & $46(0.8)$ \\
& CPU & 0.0006 & 0.0191 & 0.0062 & 0.0030 & 0.0934 \\
\hline AGBK & IT & 24 & 624 & 111 & 251 & 59 \\
& CPU & 0.0004 & 0.0134 & 0.0035 & 0.0018 & 0.0388 \\
SU-R & & 1.2246 & 1.1417 & 1.0360 & 1.2123 & 1.0771 \\
SU-A & & 2.0521 & 1.6324 & 1.8407 & 1.9953 & 2.5934 \\
\hline
\end{tabular}


relaxed parameter $\lambda_{\text {opt }}$ compared with that of GBK algorithm for different sparse systems (1) with coefficient matrices $\boldsymbol{A}$. The corresponding optimal relaxed parameter computed similarly in Example 4.3 is $\lambda_{\text {opt }}=0.9,1.2,0.9,1.2$ and 0.8, respectively. Figure 3 shows the plot of RSE versus IT (left) or RSE versus CPU (right) of Algorithm 1 applied to solve (1) with different sparse coefficient matrix $A$ in Table 4. From Table 4, we can see that the speedup ratio SU-R can reach 1.2246 and SU-A can reach 2.5934, which shows the fast convergence of our proposed algorithm. From Figure 3, RGBK and AGBK converge much faster than GBK does on RSE versus IT (left) and RSE versus CPU (right) for sparse consistent Equation (1) with coefficient matrices $\boldsymbol{A}$ named ash958 and stat96v5 in Table 4.

Example 4.5. This example uses RGBK and AGBK to restore a computer
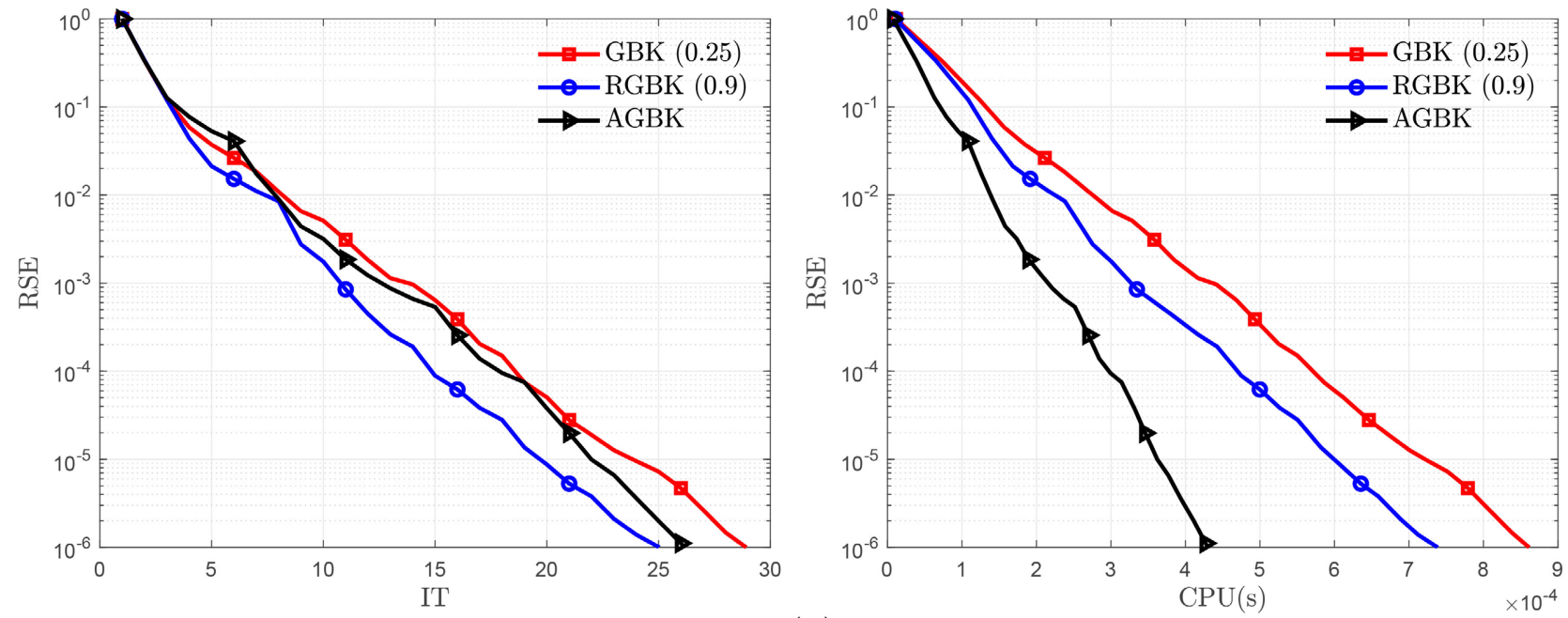

(a)
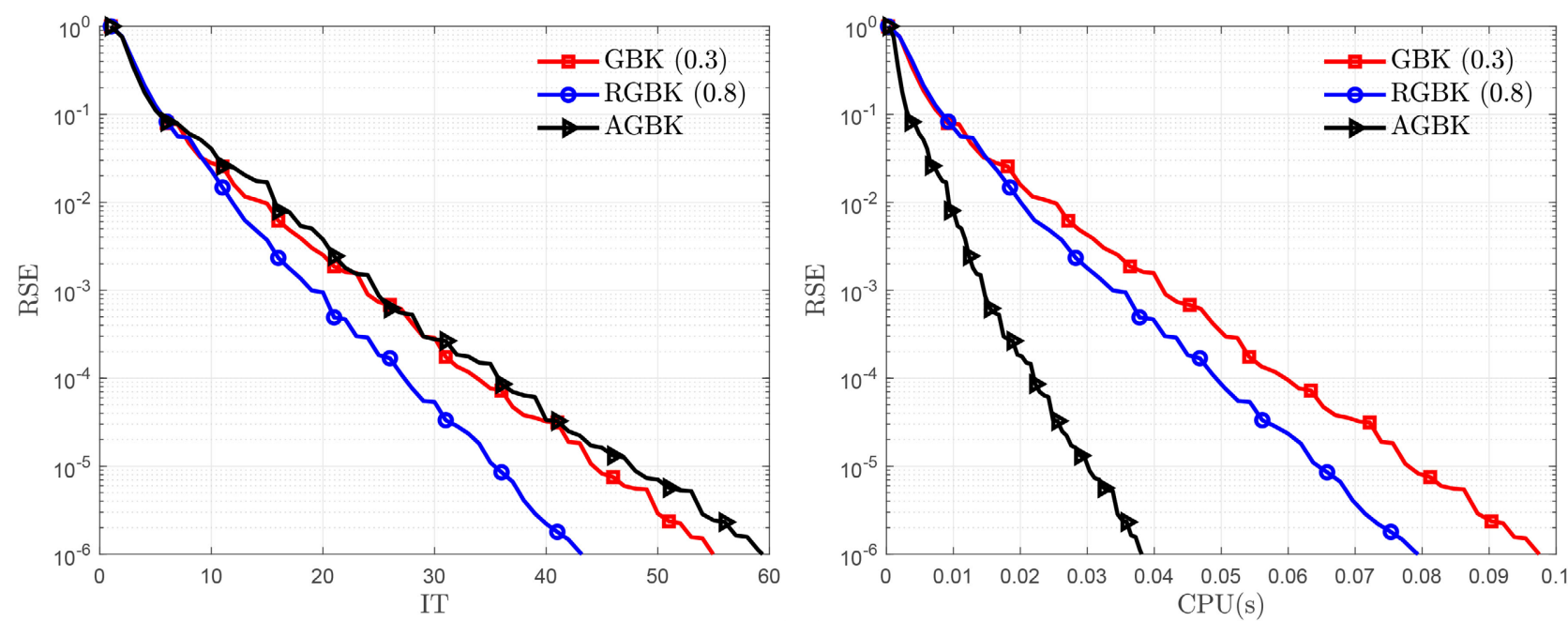

(b)

Figure 3. RSE versus IT (left) and RSE versus CPU (right) of RGBK and AGBK compared with GBK to solve consistent Equation (1) with sparse coefficient matrix $\boldsymbol{A}$ named ash958 (a) stat96v5 (b) in Table 3. (a) IT (left) and CPU (right) vesue ash958; (b) IT (left) and CPU (right) vesue stat96v5. 
tomography $(C T)$ image. The MATLAB function paralleltomo $(N, \theta, p)$ from Algebraic Iterative Reconstruction (ART) package in [26], which generates a large sparse matrix $\boldsymbol{A}$ and the exact solution $x_{*}$ is used, where $N=70$, $\theta=0^{\circ}: 0.7: 178^{\circ}$ and $p=70$, then the size of $A$ is $17,850 \times 4900$. We compute $b$ by $b=A x_{*}$, RGBK and AGBK are used to recover $x_{*}$ from $b$ and compared with the GBK method.

Table 5 reports the IT and CPU(s), and RSE of RGBK and AGBK compared with GBK for overdetermined consistent sparse matrix, where RSE $\leq 10^{-6}$. Figure 4 shows the recovered images by GBK, RGBK and AGBK together with the original image.

It can be seen from Table 5 that RGBK(1.3) and AGBK obtain lower IT and $\mathrm{CPU}(\mathrm{s})$ than $\mathrm{GBK}(0.2)$ for restoring $C T$ images, which show that RGBK and AGBK converge faster than GBK dose if the parameter $\lambda$ is selected appropriately.

Table 5. GBK, RGBK and AGBK for reconstruction of CTimage.

\begin{tabular}{cccc}
\hline Method & IT & CPU(s) & RSE \\
\hline GBK(0.2) & 863 & 5.6002 & $9.8576 \mathrm{e}-07$ \\
RGBK $(1.3)$ & 753 & 5.0474 & $8.3409 \mathrm{e}-07$ \\
AGBK & 787 & 1.9918 & $9.5513 \mathrm{e}-07$ \\
\hline
\end{tabular}

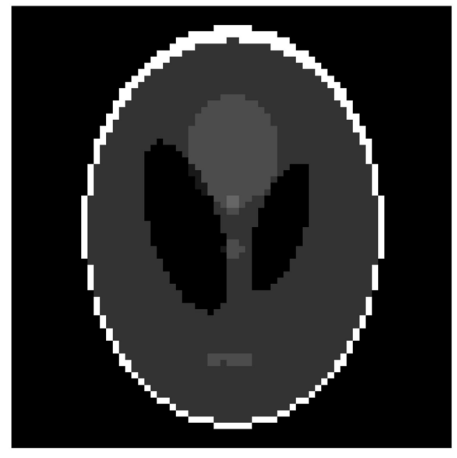

(a)

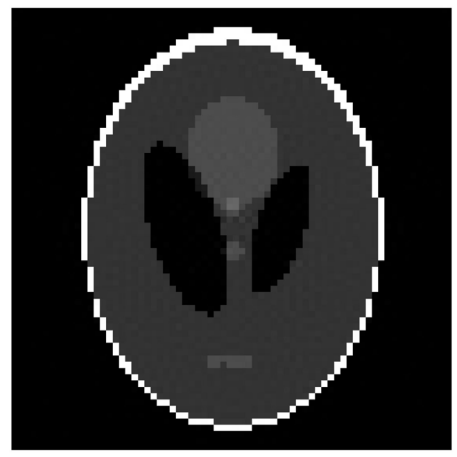

(c)

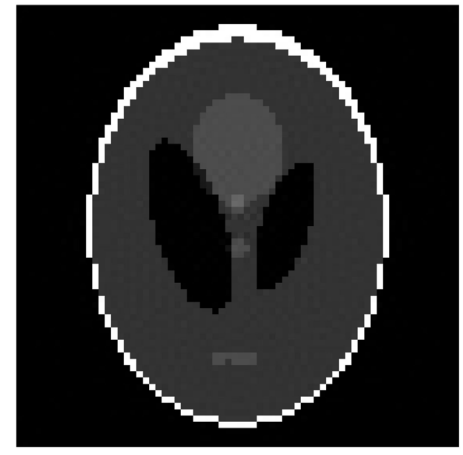

(b)

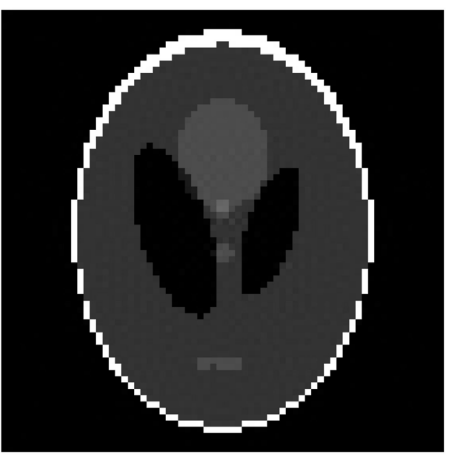

(d)

Figure 4. The original "phantom" image (a), the recovered images by GBK (b), RGBK(1.3) (c) and AGBK (d). 


\section{Conclusion}

We present a relaxed GBK algorithm abbreviated as RGBK for solving large consistent linear systems. The RGBK method extends the GBK method in [17]. The convergence is provided and a method is provided to determine an optimal relaxed parameter for the RGBK method. In addition, AGBK effectively accelerates the convergence of RGBK in running time. The examples for different cases show the advantage of the proposed RGBK and AGBK methods as long as the optimal relaxation parameter $\lambda_{\text {opt }}$ is determined.

\section{Acknowledgements}

The authors thank the reviewers for providing some helpful comments. Research by Y.L. was partially supported by The Innovation Fund of Postgraduate, Sichuan University of Science \& Engineering (grant y2021101), Research by F.Y. was partially supported by the Opening Project of Sichuan Province University Key Laboratory of Bridge Non-destruction Detecting and Engineering Computing (grant 2020QZJ03) and SUSE (grant 2019RC09, 2020RC25) and research by G.H. was supported in part by Application Fundamentals Foundation of STD of Sichuan (grant 2020YJ0366).

\section{Conflicts of Interest}

The authors declare no conflicts of interest regarding the publication of this paper.

\section{References}

[1] Niu, Y.Q. and Zheng, B. (2020) A Greedy Block Kaczmarz Algorithm for Solving Large-Scale Linear Systems. Applied Mathematics Letters, 104, Article ID: 106294. https://doi.org/10.1016/j.aml.2020.106294

[2] Kaczmarz, S. (1937) Angenäherte auflösung von systemen linearer Gleichungen. Bulletin International de 1 Académie Polonaise des Sciences et des Lettres, 35, 355-357.

[3] Gordon, R., Bender, R. and Herman, G. (1970) Algebraic Reconstruction Techniques (ART) for Three-Dimensional Electron Microscopy and X-Ray Photography. Journal of Theoretical Biology, 29, 471-481. https://doi.org/10.1016/0022-5193(70)90109-8

[4] Censor, Y. (1988) Parallel Application of Block-Iterative Methods in Medical Imaging and Radiation Therapy. Mathematical Programming, 42, 307-325. https://doi.org/10.1007/BF01589408

[5] Byrne, C. (2004) A Unified Treatment of Some Iterative Algorithms in Signal Processing and Image Reconstruction. Inverse Problems, 20, 103-120. https://doi.org/10.1088/0266-5611/20/1/006

[6] Herman, G.T. (2009) Fundamentals of Computerized Tomography: Image Reconstruction from Projections. 2nd Edition, Springer, Dordrecht.

[7] Elble, J.M. and Sahinidis, N.V. and Vouzis, P. (2010) GPU Computing with Kaczmarz and Other Iterative Algorithms for Linear Systems. Parallel Computing, 36, 215-231. https://doi.org/10.1016/j.parco.2009.12.003

[8] Eldar, Y.C. and Needell, D. (2011) Acceleration of Randomized Kaczmarz Method 
via the Johnson-Lindenstrauss Lemma. Numerical Algorithms, 58, 163-177. https://doi.org/10.1007/s11075-011-9451-Z

[9] Needell, D. and Tropp, J.A. (2014) Paved with Good Intentions: Analysis of a Randomized Block Kaczmarz Method. Linear Algebra and Its Applications, 441, 199-221. https://doi.org/10.1016/j.laa.2012.12.022

[10] Zouzias, A. and Freris, N. (2012) Randomized Extended Kaczmarz for Solving Least Squares. SIAM Journal on Matrix Analysis and Applications, 34, 773-793. https://doi.org/10.1137/120889897

[11] Briskman, J. and Needell, D. (2015) Block Kaczmarz Method with Inequalities. Journal of Mathematical Imaging and Vision, 52, 385-396. https://doi.org/10.1007/s10851-014-0539-7

[12] Ma, A., Needell, D. and Ramda, A.S. (2015) Convergence Properties of the Randomized Extended Gauss-Seidel and Kaczmarz Methods. SIAM Journal on Matrix Analysis and Applications, 36, 1590-1604. https://doi.org/10.1137/15M1014425

[13] Needell, D., Zhao, R. and Zouzias A. (2015) Randomized Block Kaczmarz Method with Projection for Solving Least Squares. Linear Algebra and its Applications, 484, 322-343. https://doi.org/10.1016/j.laa.2015.06.027

[14] Bai, Z.Z. and Wu, W.T. (2019) On Partially Randomized Extended Kaczmarz Method for Solving Large Sparse Overdetermined Inconsistent Linear Systems. Linear Algebra and Its Applications, 578, 225-250. https://doi.org/10.1016/j.laa.2019.05.005

[15] Du, K. (2019) Tight Upper Bounds for the Convergence of the Randomized Extended Kaczmarz and Gauss-Seidel Algorithms. Numerical Linear Algebra with Applications, 26, e2233. https://doi.org/10.1002/nla.2233

[16] Richtarik, P. and Takavc, M. (2020) Stochastic Reformulations of Linear Systems Algorithms and Convergence Theory. SIAM Journal on Matrix Analysis and Applications, 41, 487-524. https://doi.org/10.1137/18M1179249

[17] Strohmer, T. and Vershynin, R. (2009) A Randomized Kaczmarz Algorithm with Exponential Convergence. Journal of Fourier Analysis and Applications, 15, 262-278. https://doi.org/10.1007/s00041-008-9030-4

[18] Zhang, J.J. (2018) A New Greedy Kaczmarz Algorithm for the Solution of Very Large Linear Systems. Applied Mathematics Letters, 91, 207-212.

https://doi.org/10.1016/j.aml.2018.12.022

[19] Bai, Z.Z. and Wu, W.T. (2018) On Greedy Randomized Kaczmarz Method for Solving Large Sparse Linear Systems. SIAM Journal on Scientific Computing, 40, A592-A606. https://doi.org/10.1137/17M1137747

[20] Bai, Z.Z. and Wu, W.T. (2018) On Relaxed Greedy Randomized Kaczmarz Methods for Solving Large Sparse Linear Systems. Applied Mathematics Letters, 83, 21-26. https://doi.org/10.1016/j.aml.2018.03.008

[21] Björck, A. (1996) Numerical Methods for Least Squares Problems. SIAM, Philadelphia. https://doi.org/10.1137/1.9781611971484

[22] Gower, R.M. and Richtárik, P. (2015) Randomized Iterative Methods for Linear Systems. SIAM Journal on Matrix Analysis and Applications, 36, 1660-1690. https://doi.org/10.1137/15M1025487

[23] Kashkari, B. and Alqarni, S. (2019) Two-Step Hybrid Block Method for Solving Nonlinear Jerk Equations. Journal of Applied Mathematics and Physics, 7, 1897-1910. https://doi.org/10.4236/jamp.2019.78130

[24] Duromola, M. and Momoh, A. (2019) Hybrid Numerical Method with Block Extension for Direct Solution of Third Order Ordinary Differential Equations. American 
Journal of Computational Mathematics, 9, 68-80.

https://doi.org/10.4236/ajcm.2019.92006

[25] Davis, T.A. and Hu, Y. (2011) The University of Florida Sparse Matrix Collection. ACM Transactions on Mathematical Software, 38, 1-25. https://doi.org/10.1145/2049662.2049663

[26] Hansen, R.C. and Jorgensen, J.S (2018) AIR Tools II: Algebraic Iterative Reconstruction Methods. Numerical Algorithms, 79, 107-137.

https://doi.org/10.1007/s11075-017-0430-x 